\title{
CAD-based calibration of a 3D DIC system
}

\author{
B. Beaubier ${ }^{1,2}$, K. Lavernhe-Taillard ${ }^{1}$, G.Besnard ${ }^{1}$, S. Lavernhe ${ }^{3}$, F. Hild ${ }^{1}$ and S. Roux ${ }^{1}$ \\ ${ }^{1}$ LMT Cachan, ENS Cachan / CNRS UMR 8535 / Univ. Paris 6 / PRES UniverSud Paris \\ 61 Avenue du Président Wilson, 94235 Cachan Cedex, France \\ benoit.beaubier@Imt.ens-cachan.fr \\ 2 PSA Peugeot Citroën, Centre Technique de Vélizy B \\ Zone aéronautique Louis Bréguet \\ 78140 VELIZY VILLACOUBLAY, France \\ ${ }^{3}$ LURPA, ENS Cachan / Université Paris-Sud 11 \\ 61 Avenue du Président Wilson, 94235 Cachan Cedex, France
}

\begin{abstract}
The aim of this study is to predict the strains induced by welding-brazing of automotive thin metal sheets using finite elements simulations. In order to validate the simulations, experimental tests on industrial parts are carried out. One goal of these tests is to determine 3D surface displacement fields during welding. The measurement technique chosen for these tests is 3D surface DIC (stereocorrelation). To take into account the specificity of these tests (object size about one square meter) a new calibration method of the stereovision system has been developed. Currently, calibration methods are based on the knowledge of the calibration target geometry and its projections onto both right and left camera frames. In the approach proposed herein, the calibration is performed directly on the measured part, which is modeled by a Bézier patch. A first step consists in finding the best 3D (CAD surface) to 2D (camera picture) transformation.
\end{abstract}

\section{INTRODUCTION}

3D surface Digital Image Correlation (stereocorrelation) is a measurement technique of 3D surface displacement fields by comparison between two reference and two deformed pictures. Image registration is possible thanks to a random pattern deposited onto the observed part, here, an automotive roof. The method is based on a stereovision process, which consists in determining 3D points from a pair of pictures with two different cameras, at different fixed angles. The 3-dimensional shape reconstruction is carried out thanks to a calibration procedure, which is the subject of this paper. The calibration consists in the determination of intrinsic parameters (internal parameters of cameras) and of extrinsic parameters (angle and position of the cameras in the experimental frame).

Most of the stereovision methods use calibration targets whose geometry is known. Calibration target geometries are numerous, the most used objects are planar. To calibrate a stereocorrelation device with this kind of target, many pairs of pictures of the calibration target in various positions and angles are required. Moreover, this method is very dependent on the geometric quality of the calibration target [1,2]. Besnard et al. [3], among others, have used 3D calibration targets. The chosen geometry has an open book shape. It allows the calibration to be performed with only one pair of pictures thanks to the 3D geometry. This calibration implementation is easier than the one using planar targets. However, the target size should be similar to the observed part. Furthermore, it is more difficult to ensure the geometric quality of (large) calibration target [2]. Other studies propose "autocalibration" methods in order to calibrate stereovision systems without a priori knowledge of the observed part. However, uncertainties about dimensions of the reconstructed object are not always satisfactory [4]. 


\section{NEW APPROACH}

This paper presents a new calibration approach. The idea is to use no more calibration target, and perform directly the calibration on the measured part. An appropriate modeling of the observed part is needed. For the final application, the part of interest is an automotive thin sheet roof for witch geometry is described with freeform surfaces (as used in Computer Aided Design (CAD) models, for instance Bézier patches [5]). To prove the feasibility of such calibration method, a test part made of aluminum alloy was machined (Figure 1). It was created as a Bézier patch in CatiaV5 and milled on a 3 axis high speed machine (HSM). To minimize deviations induced by milling between the CAD model and the actual geometry, machining tolerance and scallop height were required to be less than $1 \mu \mathrm{m}$. Polynomial interpolation was also used through the computer numerical control (CNC) options of the HSM to avoid chordal errors and to improve the follow-up.

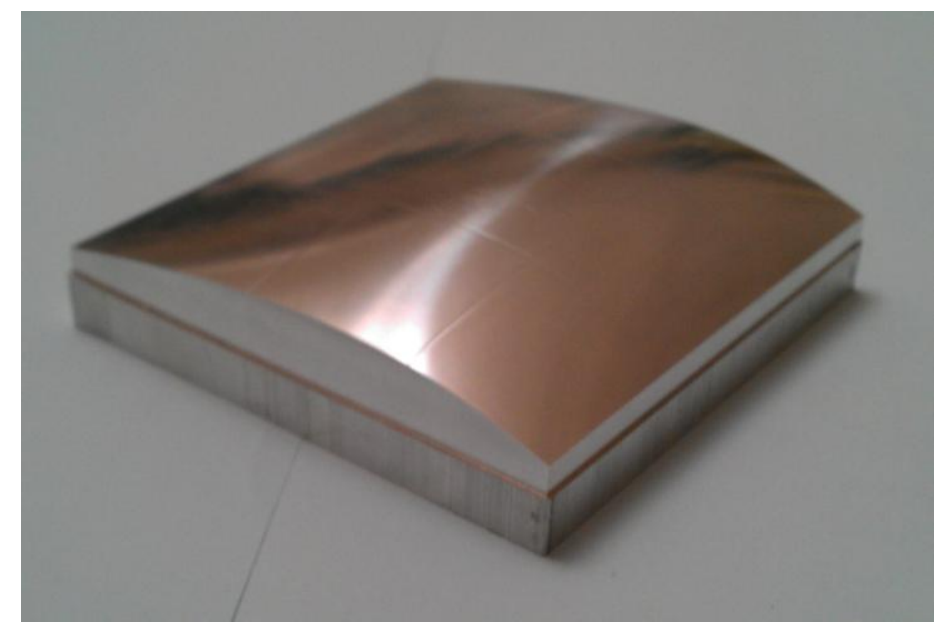

Figure 1: Part made of aluminum alloy and machined with an HSM.

The parametric description of the surface then reads [5]

$$
S(u, v)=\sum_{i=0}^{m} \sum_{j=0}^{n} B_{i m}(u) B_{j n}(v) P_{i j}, \text { with }(u, v) \in[0,1]^{2}
$$

where $u$ and $v$ are the parametric coordinates, $B_{i m}$ and $B_{j n}$ Bernstein's polynomial of degree $m$ and $n$

$$
B_{i m}(u)=\frac{m !}{i !(m-i) !} u^{i}(1-u)^{m-i}
$$

and $P_{i j}$ denote the control vertices. In the present case, the milled part is modeled by a Bézier patch of degree $(3,3)$, i.e. with 16 vertices. In Figure 2, the red part is the Bézier patch corresponding to the CAD model of the studied part, and the blue lines define the control polygon. 


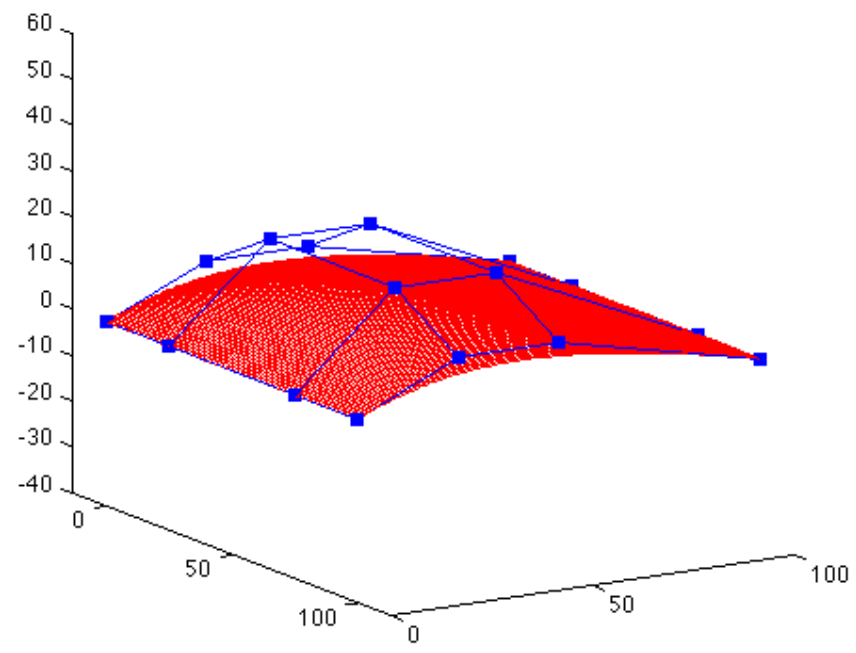

Figure 2: Theoretical Bézier patch of the studied part (Figure 1). Dimensions are in millimeters. In red, the theoretical surface and in blue the corresponding control polygon of the Bézier patch.

\section{ADVANTAGES}

Such a calibration method has many advantages. First, as no calibration target is needed (i.e., calibration is directly performed from the pair of pictures of the object) the practical implementation of the calibration method is easier. The second advantage is the basis used to express the 3D shape. The choice of a geometry defined by a Bézier surface allows us to use a global approach to DIC [6]. In the present case, an integrated-type of approach is chosen [7]. For the latter, the sought displacement field requires a small number of parameters, thereby regularizing the analysis. The chosen description of the geometry is suited for this kind of approach. With this point of view, the present implementation departs from that proposed by Besnard et al. [3], who used a global approach for the 3D matching, but it still yielded a cloud of 3D independent points.

\section{PRINCIPLE OF CALIBRATION SYSTEM}

The calibration principle is presented in Figure 3. First, an initialization is performed with the same pair of images (left and right) of the part taken by the two cameras in their chosen position. The user selects at least 6 points pairs $\left(x_{i}, y_{i}\right)$ on the right and left pictures. The three-dimensional coordinates $\left(X_{i}, Y_{i}, Z_{i}\right)$ of these points have to be known on the CAD model. After rewriting system (2), a singular value decomposition [8] gives a first estimation of right $\tilde{\mathbf{M}}_{r}$ and left $\tilde{\mathbf{M}}_{l}$ transformation matrices. In a second step, the theoretical surface $S(u, v)$ is then projected onto the right and to left pictures using the matrices previously determined. The projections are interpolated on right and left pictures. Matrix parameters $m_{i j}$ are then updated by using an integrated correlation algorithm. Final values of $m_{i j}$ are obtained after convergence of the correlation procedure.

The apparent displacement field has to be expressed on a reduced judiciously chosen kinematic basis,. The mathematical formulation of the problem is presented hereafter. The relationships between three-dimensional points and their (right and left) projections are given by

$$
\left(\begin{array}{c}
l_{l} x_{l} \\
l_{l} y_{l} \\
l_{l}
\end{array}\right)=\mathbf{M}_{l}\left(\begin{array}{c}
X \\
Y \\
Z \\
1
\end{array}\right) \quad \text { and } \quad\left(\begin{array}{c}
l_{r} x_{r} \\
l_{r} y_{r} \\
l_{r}
\end{array}\right)=\mathbf{M}_{r}\left(\begin{array}{c}
X \\
Y \\
Z \\
1
\end{array}\right)
$$


where $x_{l}$ and $y_{l}$ are pixel coordinates on the left picture, $x_{r}$ and $y_{r}$ pixel coordinates of the right picture, $l_{l}$ and $l_{r}$ the scale factors, and $X, Y, Z, 1$ homogeneous coordinates of the considered point of the CAD geometry.

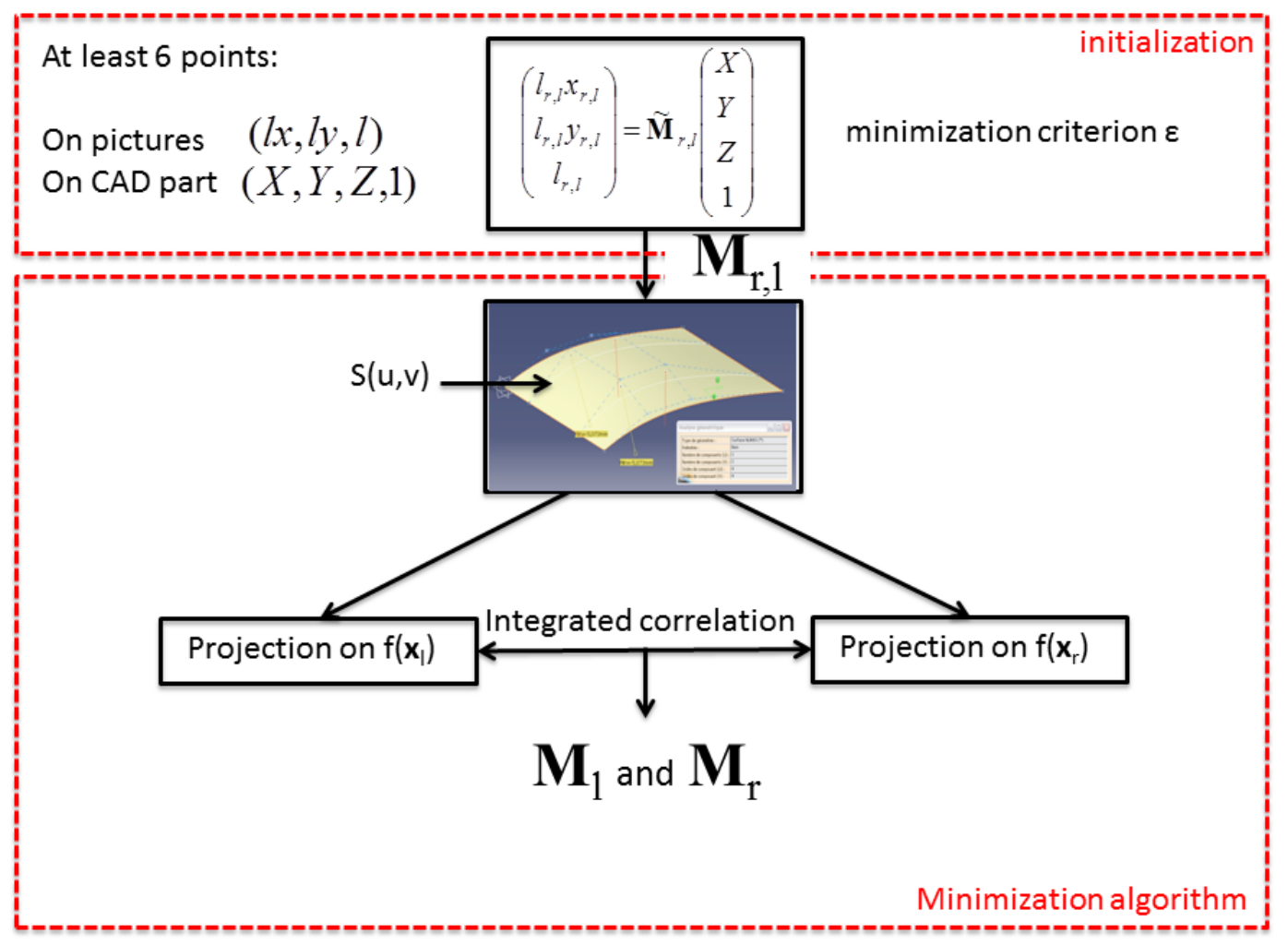

Figure 3: Principle of the calibration procedure.

These equations are rewritten as

$$
\left\{\begin{array}{l}
x_{l, r}=m_{11}^{l, r} X+m_{12}^{l, r} Y+m_{13}^{l, r} Z+m_{14}^{l, r} \\
y_{l, r}=m_{21}^{l, r} X+m_{22}^{l, r} Y+m_{23}^{l, r} Z+m_{24}^{l, r} \\
l_{l, r}=m_{31}^{l, r} X+m_{32}^{l, r} Y+m_{33}^{l, r} Z+m_{34}^{l, r}
\end{array}\right.
$$

In order to solve Equations (2), $x_{l, r}$ and $y_{l, r}$ are divided by $l_{l, r}$ so that

$$
\left\{\begin{array}{l}
x_{l, r}=\frac{m_{1 i}^{l, r} \bar{X}_{i}}{l_{l, r}} \\
y_{l, r}=\frac{m_{2 i}^{l, r} \bar{X}_{i}}{l_{l, r}}
\end{array}\right.
$$

where $\overline{\mathrm{X}_{\mathrm{i}}}=(X, Y, Z, 1)$ are the homogeneous coordinates. The problem has 24 unknowns to be determined (i.e., 24 components of matrices $\mathbf{M}_{\mathbf{r}}$ and $\mathbf{M}_{\mathbf{l}}$ ). Consequently, we have to use a kinematic basis with at most 24 components. Only 22 are considered in practice since the matrix components are determined up to a multiplicative constant. The 'kinematic' basis allows us to determine matrix parameters $m_{i j}$ directly. In order to determine the basis the coordinate sensitivity is expressed as a function of the matrix parameters $m_{i j}$ 


$$
d \mathbf{x}_{l, r}=d \mathbf{x}_{l, r}\left(\tilde{m}_{i j}\right)+\frac{\partial d \mathbf{x}_{l, r}}{\partial m_{i j}} d m_{i j}
$$

where $d \mathbf{x}_{l, r}$ denote the apparent displacements in the left and right pictures induced by a change in the component of the transformation matrices. The images shot by left and right cameras are respectively labeled as $f\left(\mathbf{x}_{l}\right)$ and $g\left(\mathbf{x}_{r}\right)$. An image is a matrix with a gray level value for each pixel coordinate $\mathbf{x}$. The conservation of gray level reads

$$
f\left(\mathbf{x}_{l}+d \mathbf{x}_{l}\right)=g\left(\mathbf{x}_{r}+d \mathbf{x}_{r}\right)
$$

Contrary to classical DIC approaches in which the reference picture does not change during the iterations of the minimization procedure, the two pictures are updated at each iteration. The linearized conservation law reads

$$
f\left(\mathbf{x}_{l}\right)+\nabla f \cdot d \mathbf{x}_{l}=g\left(\mathbf{x}_{r}\right)+\nabla g \cdot d \mathbf{x}_{r}
$$

The problem thus amounts to minimizing the following residual

$$
T=\int\left(f\left(\mathbf{x}_{l}\right)-g\left(\mathbf{x}_{r}\right)+\nabla f \cdot d \mathbf{x}_{l}-\nabla g \cdot d \mathbf{x}_{r}\right)^{2} d u d v
$$

with respect to the unknown $m_{i j}$ parameters. A multiscale iterative procedure is implemented to solve this problem [6].

\section{CALIBRATION TEST}

Measurements have been carried out on the part shown in Figure 1, when black and white paint was sprayed onto the external surface. Cameras used herein were Canon EOS $40 \mathrm{D}$ with a CMOS sensor of definition $3888 \mathrm{x}$ 2592 pixels (in RGB). After transformation into gray levels, the final definition of the pictures is $1944 \times 1296$ pixels. The two analyzed pictures are shown in Figure 4.

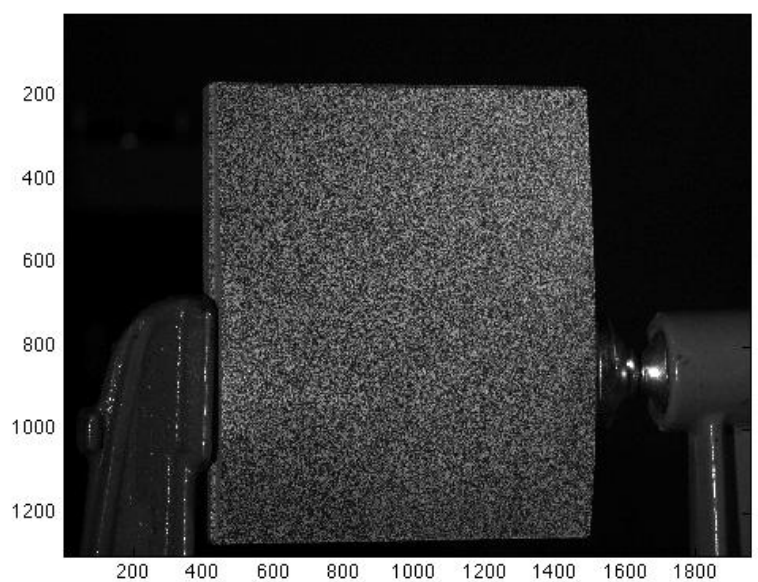

Left picture $f\left(\mathbf{x}_{l}\right)$

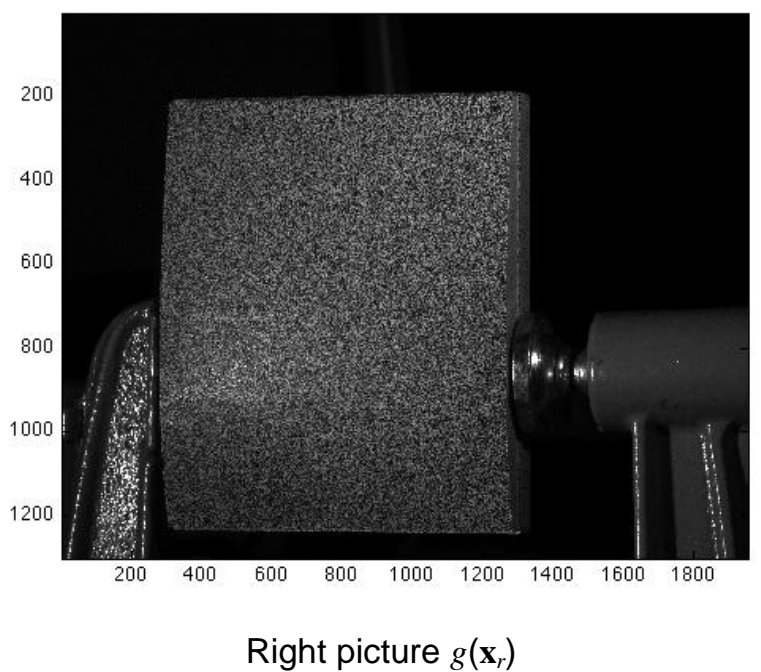

Right picture $g\left(\mathbf{x}_{r}\right)$

Figure 4: Pictures taken by the left and right cameras.

In the correlation algorithm, the dimension of the parametric space $(u, v)$ is $1000 \times 1000$ pixels. Figure 5 shows the map of the correlation residuals (i.e. difference $(f-g)$ in the $(u, v)$ plane) at the end of the calibration procedure. This map accounts for both correlation and geometric errors. It is worth emphasizing that up to now, the transformation matrix was determined from the theoretical CAD geometry. No large deviation from the CAD model is observed in Figure 5. The largest difference is due to a spurious specular reflection of light onto the surface captured by the 
right camera, but not the left one. The dimensionless residuals were $2.1 \%$ as compared to the initial residual obtained with the manual pointing of characteristic points.

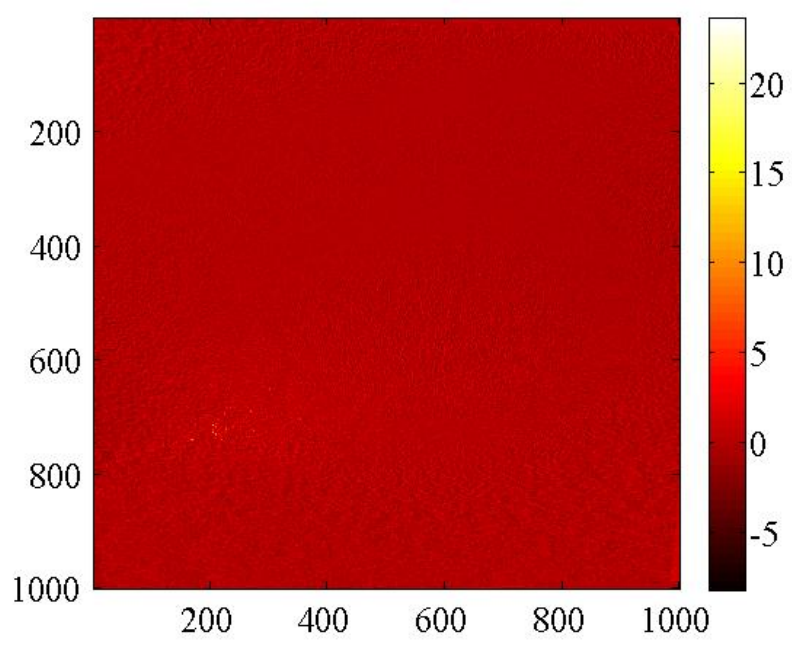

Figure 5: Map of the final correlation residual expressed in percent of the dynamic range of the analyzed pictures (mean value $\eta=2.1 \%$ )

\section{CONCLUSIONS}

It has been shown that it is possible to calibrate a stereovision setup (i.e., components of the transformation matrix) using a CAD model of the observed part. A global and integrated correlation scheme was implemented. Small correlation residuals were found, thereby validating the proposed procedure. The next step is to account for optical distortions to complete the calibration of the stereovision system. The last step is the application to the analysis of an automotive roof and its subsequent deformation during weld brazing.

\section{REFERENCES}

[1] M. A. Sutton, J. J. Orteu, H. W. Schreier, Image Correlation for Shape, Motion and Deformation Measurements: Basic Concepts, Theory and Applications. Springer, New York, NY (USA), 2009.

[2] D. Garcia, J.J. Orteu, L. Penazzi, A combined temporal tracking and stereo-correlation technique for accurate measurement of 3D displacements: application to sheet metal forming. JOURNAL OF MATERIALS PROCESSING TECHNOLOGY, 125-126: 736-742, 2002.

[3] G. Besnard, J.M Lagrange, F. Hild, S. Roux, C. Voltz, Characterization of necking phenomena in high speed experiments by using a single camera. EURASIP, JOURNAL ON IMAGE AND VIDEO PROCESSING, 2010(21595): 15p., 2010.

[4] A. Fusiello, Uncalibrated Euclidean reconstruction: a review. IMAGE AND VISION COMPUTING, 18: 555563, 2000.

[5] L.A. Piegl, W. Tiller, The NURBS Book. Sringer, 1995.

[6] G. Besnard, F. Hild, and S. Roux. "Finite-Element" displacement fields analysis from digital images: Application to portevin-le chatelier bands. EXPERIMENTAL MECHANICS, 46(6): 789-803, 2006.

[7] S. Roux, F. Hild, Stress intensity factor measurements from digital image correlation: post-processing and integrated approaches. INTERNATIONAL JOURNAL OF FRACTURE, 142: 51-67, 2006.

[8] J. Salvi, X. Armague, J. Battle. A comparative review of camera calibrating methods with accuracy evaluation. PATTERN RECOGNITION, 35: 1617-1635, 2002. 